Supplementary files

\title{
Computational Variation: An Under-Investigated Quantitative Variability Caused by Automated Data Processing in Untargeted Metabolomics
}

\author{
Huaxu $\mathrm{Yu}^{1}$, Ying $\mathrm{Chen}^{1}$, Tao Huan ${ }^{1 *}$ \\ ${ }^{1}$ Department of Chemistry, Faculty of Science, University of British Columbia, Vancouver \\ Campus, 2036 Main Mall, Vancouver, V6T 1Z1, BC, Canada
}

*Author to whom correspondence should be addressed:

Dr. Tao Huan

Tel: (+1)-604-822-4891

E-mail: thuan@chem.ubc.ca

Internet: https://huan.chem.ubc.ca/ 


\section{Outline}

Figure S-1. The correlation between computational variation and analytical variation.

Figure S-2. Comparison of relative standard deviation (RSD) calculated by using peak height and peak area (XCMS).

Figure S-3. Factors contributing to peak height and peak area-based quantifications in urine samples.

Figure S-4. Factors contributing to peak height and peak area-based quantifications in mouse fecal samples.

Figure S-5. Factors contributing to peak height and peak area-based quantifications in mouse gut content samples.

Figure S-6. RSD distributions for low concentration metabolomic data processed by XCMS.

Figure S-7. Comparison of different reconstitution solvent using human serum metabolome extract.

Figure S-8. RSD distributions for human serum lipidomic data acquired from RP+ mode.

Figure S-9. RSD distributions for metabolomic data processed by XCMS without optimizing parameter settings.

Figure S-10. Representative extracted ion chromatograms (EICs) of metabolites with lower RSDs using peak height or peak area-based quantification.

Figure S-11. Preference on peak height or peak area for metabolic features with consistent preference

Figure S-12. A representative example of metabolite (alpha-Ketoisovaleric acid) with increased $p$-value after correction.

Figure S-13. Manual inspection of the peak area-based quantitative results.

Table S-1. Concentration table of pooled 20 metabolite standard solution.

Table S-2. RSD comparison of using manual integration approach and automated computational approach in targeted LC-MS analysis.

Table S-3. Number of extracted metabolic features when $t=5 \%$.

Table S-4. Number of extracted metabolic features when $t=10 \%$.

Table S-5. Number of extracted metabolic features when $t=15 \%$. 
Table S-6. Number of extracted metabolic features when $t=20 \%$.

Text S-1. Metabolite extraction of human serum and urine samples.

Text S-2. LC gradient and other settings.

Text S-3. Parameter settings of mass spectrometer.

Text S-4. MS-DIAL parameter settings for data processing.

Text S-5. XCMS parameter settings for data processing (optimized).

Text S-6. XCMS parameter settings for data processing (not optimized).

Text S-7. Manual inspection in MS-DIAL. 

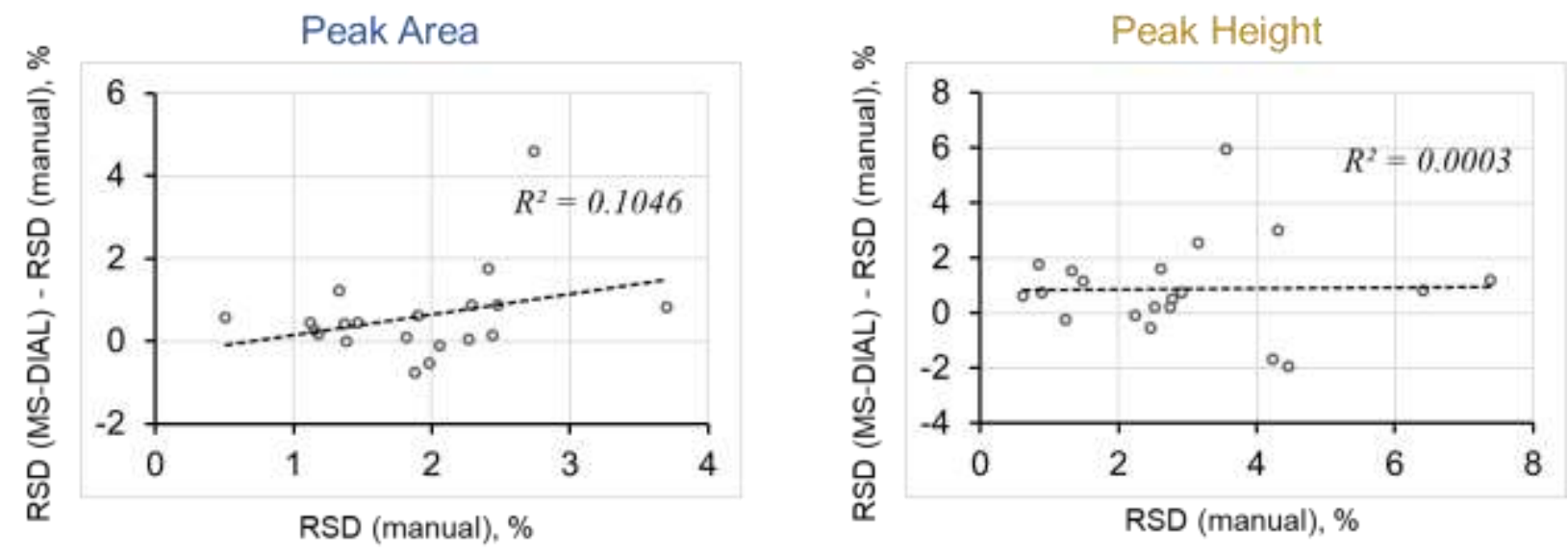

Figure S-1. The correlation between computational variation and analytical variation. The computational variations were computed by both peak area (left) and peak height (right). The analytical variation was calculated from manual integration approach. Each dot on plot represents one metabolite standard. 


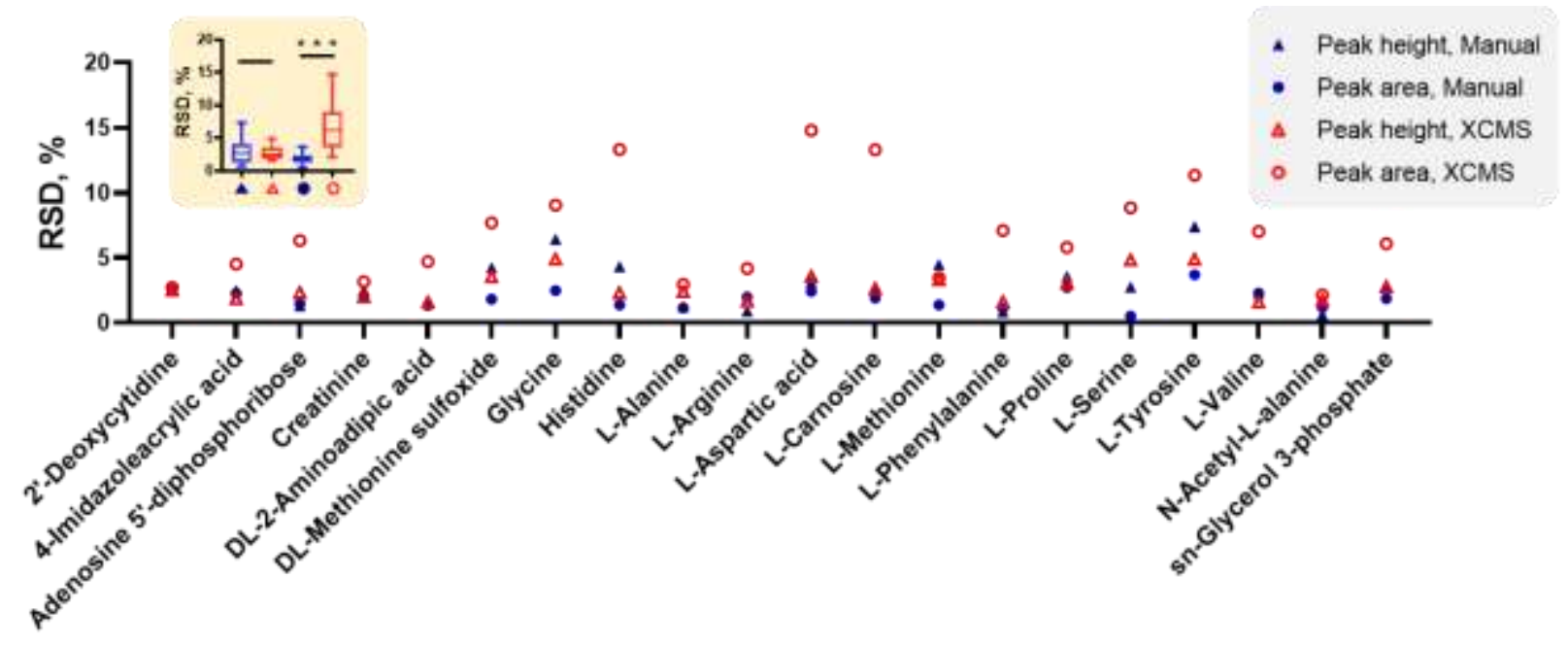

Figure S-2. Comparison of relative standard deviation (RSD) calculated by using peak height and peak area. Manual checking and data processing software (XCMS) was utilized to process the same data set. The three * means the $p$ value is lower than 0.001 , which is calculated from ${ }^{1}$ twotailed $t$ test. 


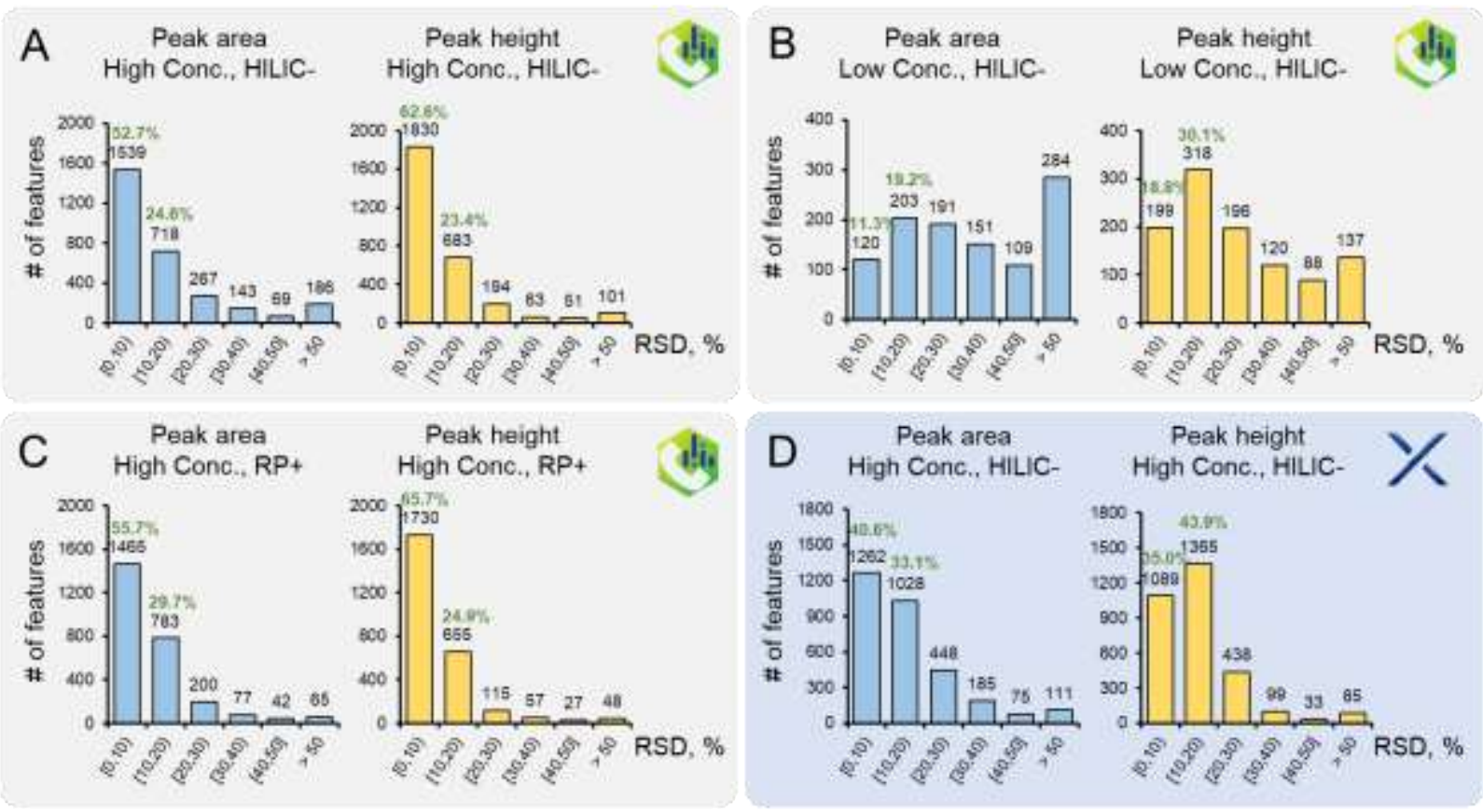

Figure S-2. Factors contributing to peak height and peak area-based quantifications in urine samples. (A) High concentration, analyzed in HILIC- mode and processed by MS-DIAL; (B) Low concentration, analyzed in HILIC- mode and processed by MS-DIAL; (C) High concentration, analyzed in RP+ mode and processed by MS-DIAL; (D); High concentration, analyzed in HILICand processed by XCMS. 

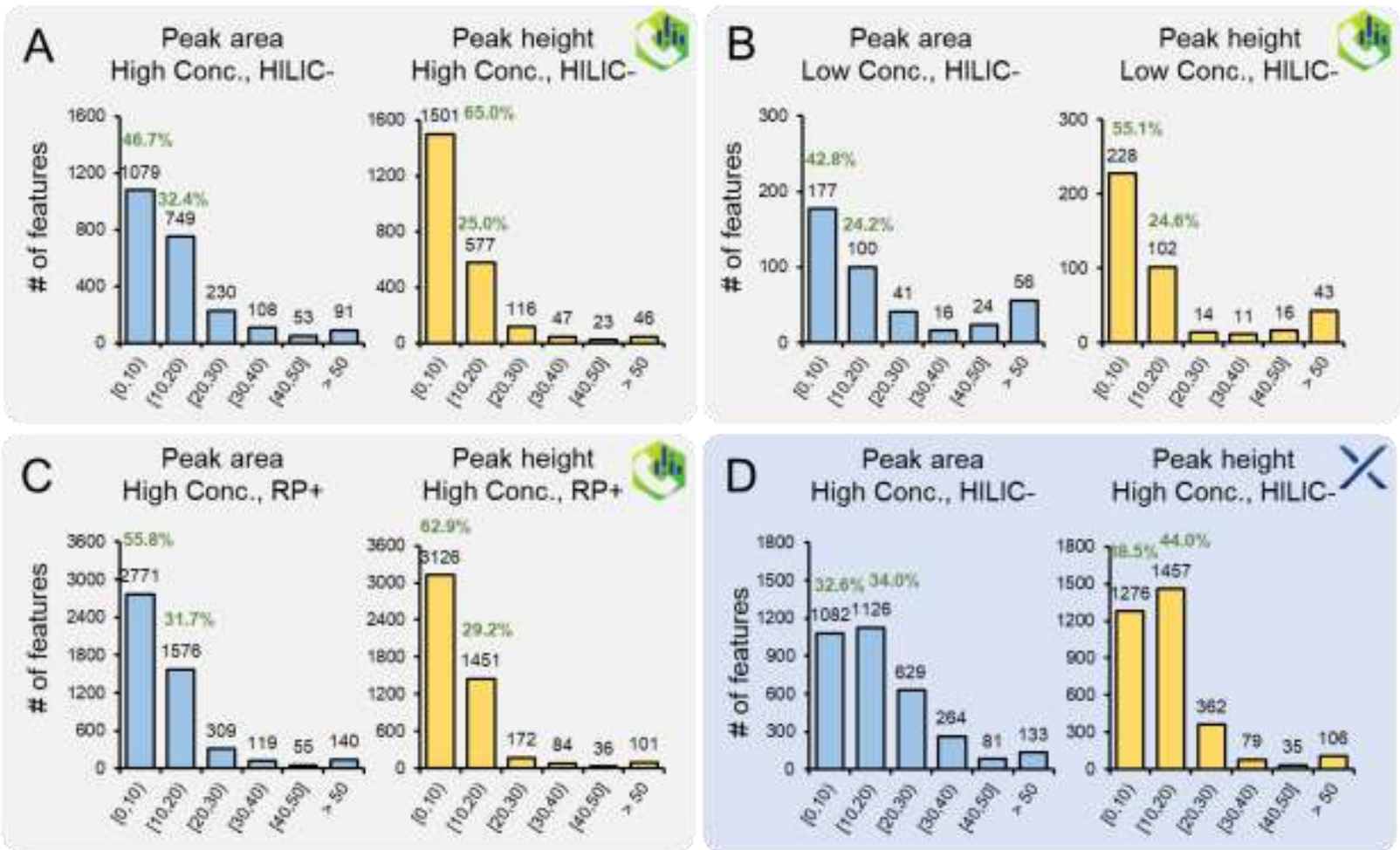

Figure S-3. Factors contributing to peak height and peak area-based quantifications in mouse fecal samples. (A) High concentration, analyzed in HILIC- mode and processed by MS-DIAL; (B) Low concentration, analyzed in HILIC- mode and processed by MS-DIAL; (C) High concentration, analyzed in RP+ mode and processed by MS-DIAL; (D); High concentration, analyzed in HILICand processed by XCMS. 


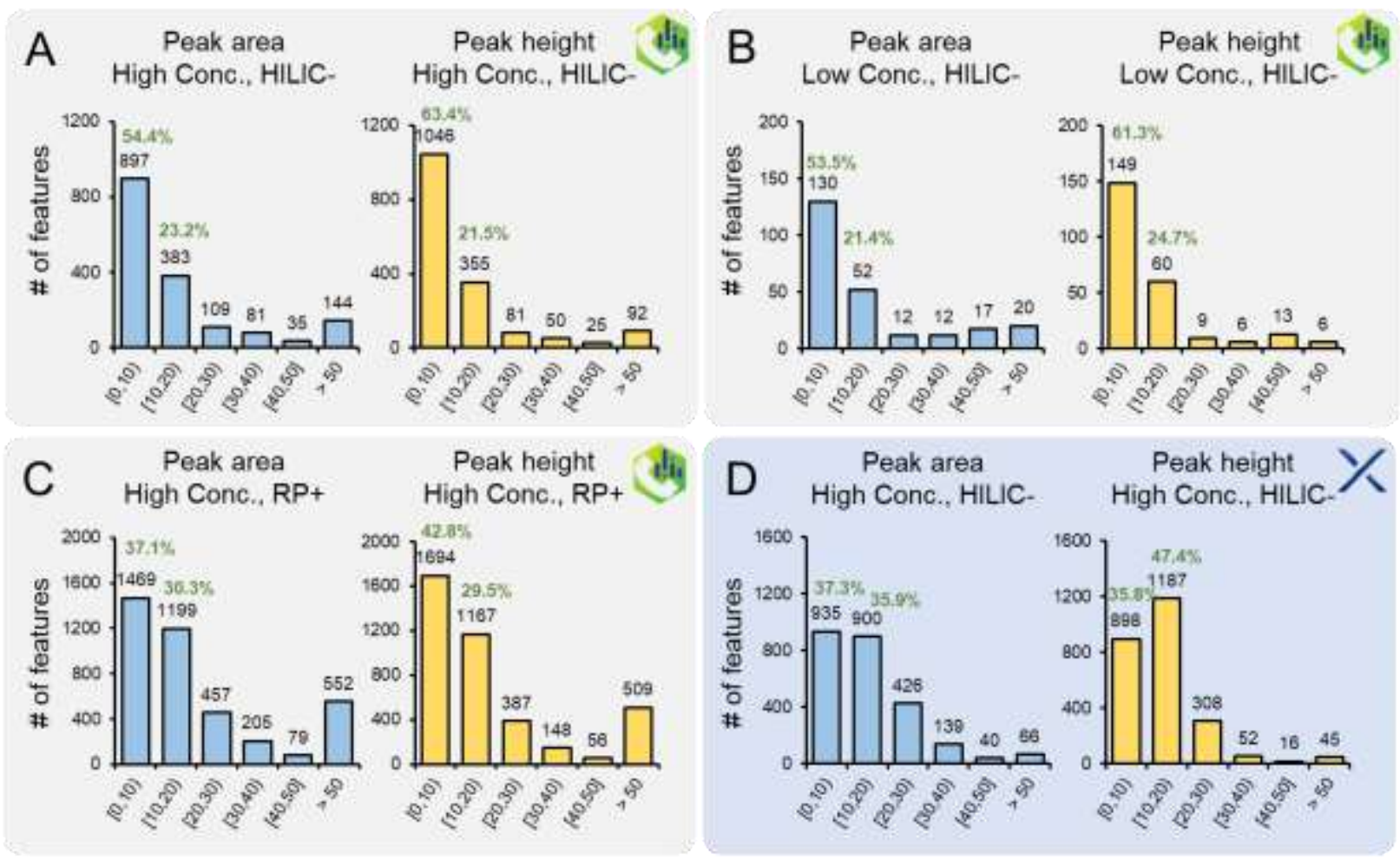

Figure S-4. Factors contributing to peak height and peak area-based quantifications in mouse gut content samples. (A) High concentration, analyzed in HILIC- mode and processed by MS-DIAL; (B) Low concentration, analyzed in HILIC- mode and processed by MS-DIAL; (C) High concentration, analyzed in RP+ mode and processed by MS-DIAL; (D); High concentration, analyzed in HILIC- and processed by XCMS. 

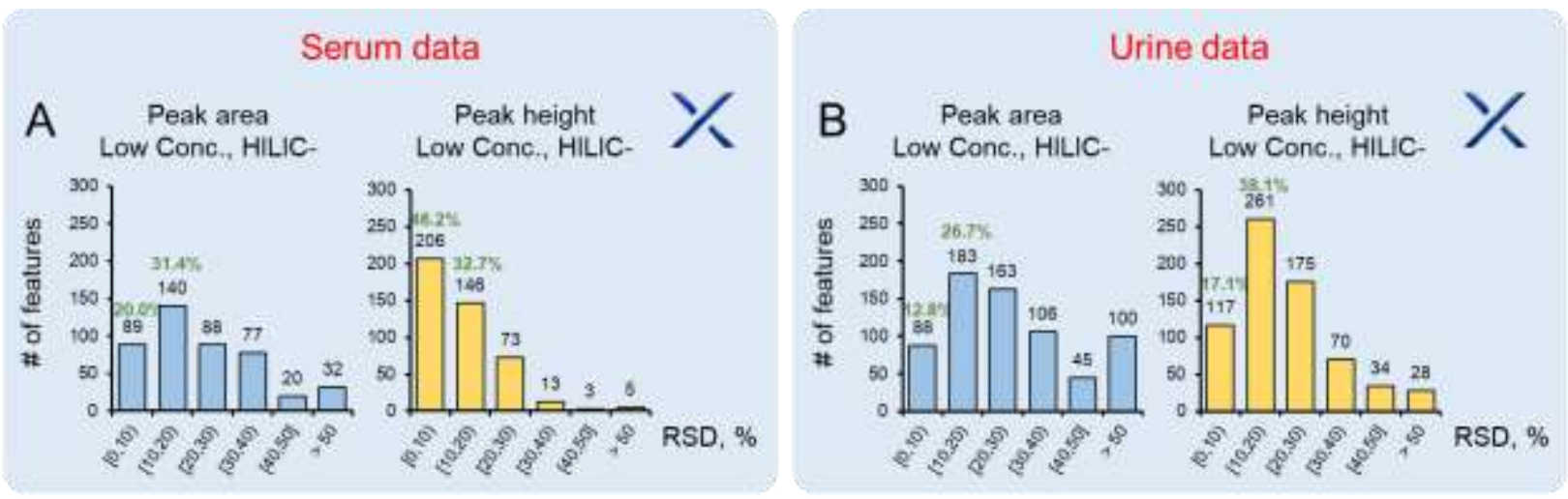

Figure S-5. RSD distributions for low concentration metabolomic data processed by XCMS. (A) Serum data; (B) Urine data. 


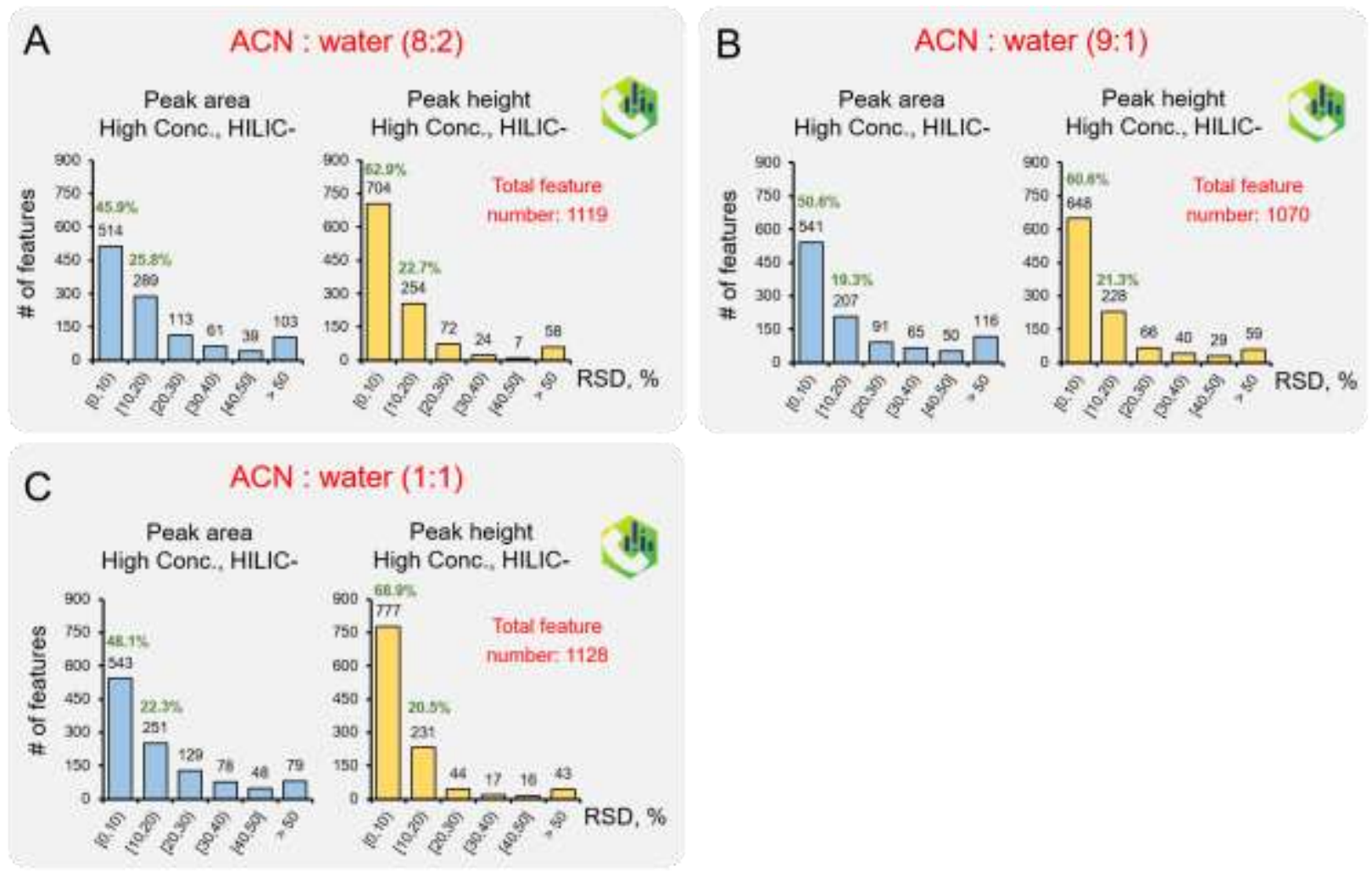

Figure S-6. Comparison of different reconstitution solvent using human serum metabolome extract. The composition of reconstitution solvents are (A) ACN:water = 8:2; (B) ACN:water = $9: 1 ;(\mathrm{C})$ ACN:water $=1: 1$. 

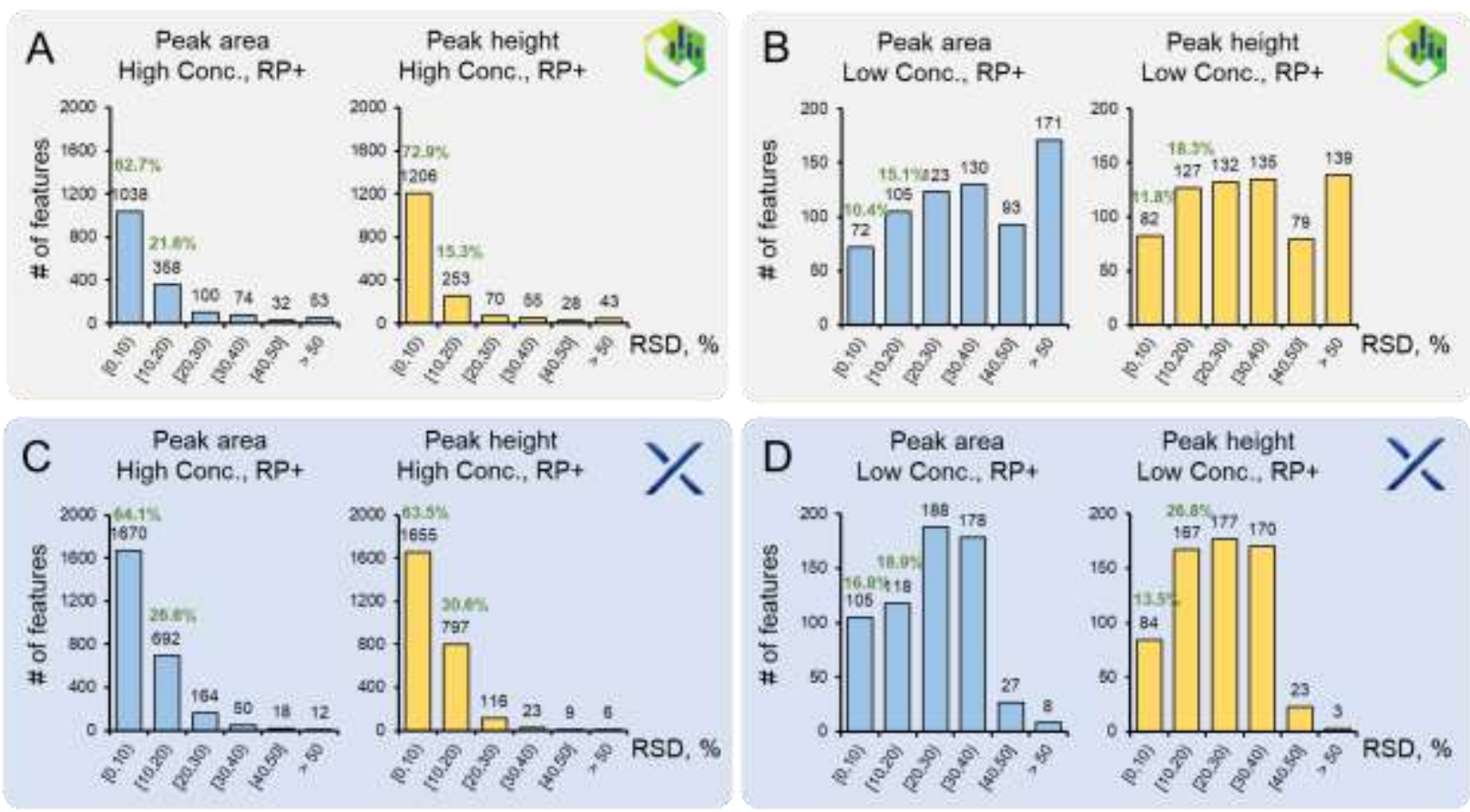

Figure S-7. RSD distributions for human serum lipidomic data acquired from RP+ mode. (A) High concentration processed by MS-DIAL; (B) Low concentration processed by MS-DIAL; (C) High concentration processed by XCMS; (D) Low concentration processed by XCMS. 

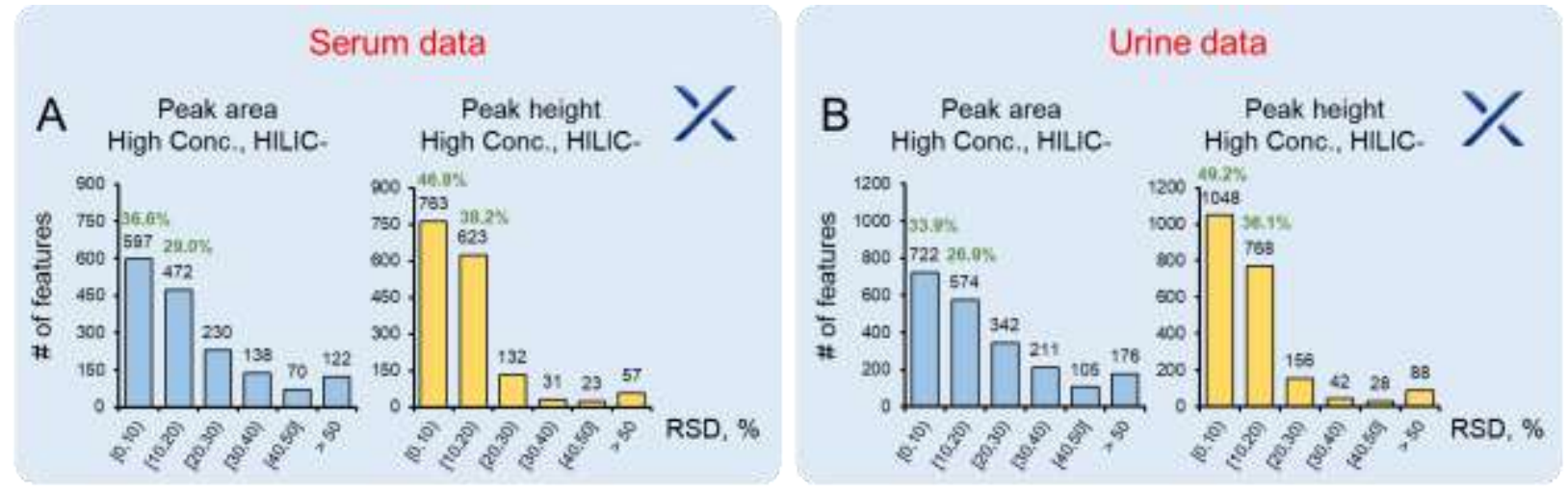

Figure S-8. RSD distributions for metabolomic data processed by XCMS without optimizing parameter settings. (A) Serum data; (B) Urine data. 

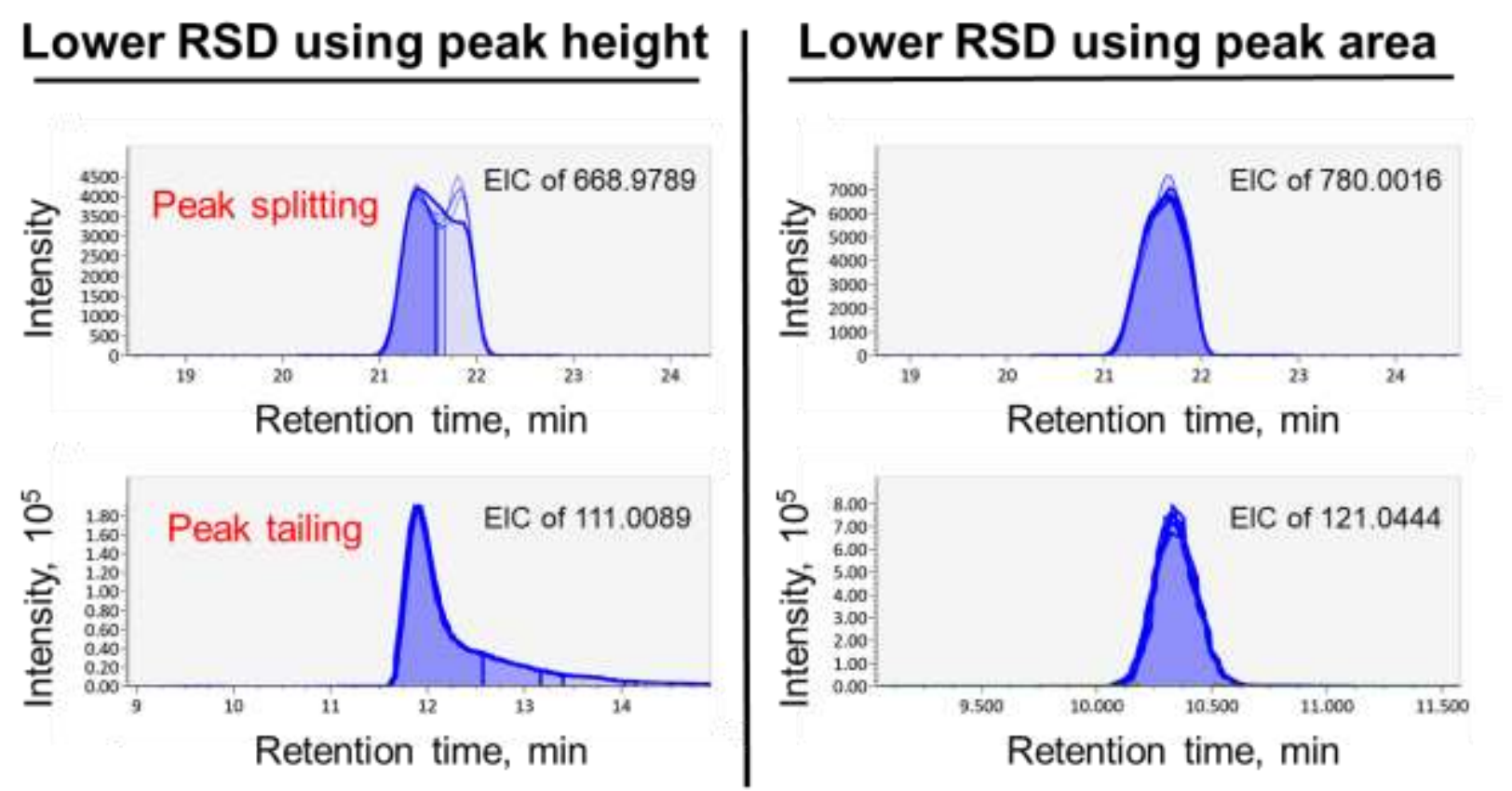

Figure S-9. Representative extracted ion chromatograms (EICs) of metabolites with lower RSDs using peak height or peak area-based quantification. Data were generated using a serum sample analyzed in HILIC- mode and processed by MS-DIAL. All five analytical replicates are presented. The colored area indicates the integrated peak area. Different lines indicate the different samples from five replicates. A bold line means that this feature is detected in a sample by local maximum algorithm. A fine line means that this feature is gap-filled in a sample since local maximum is not observed in the chromatogram. 


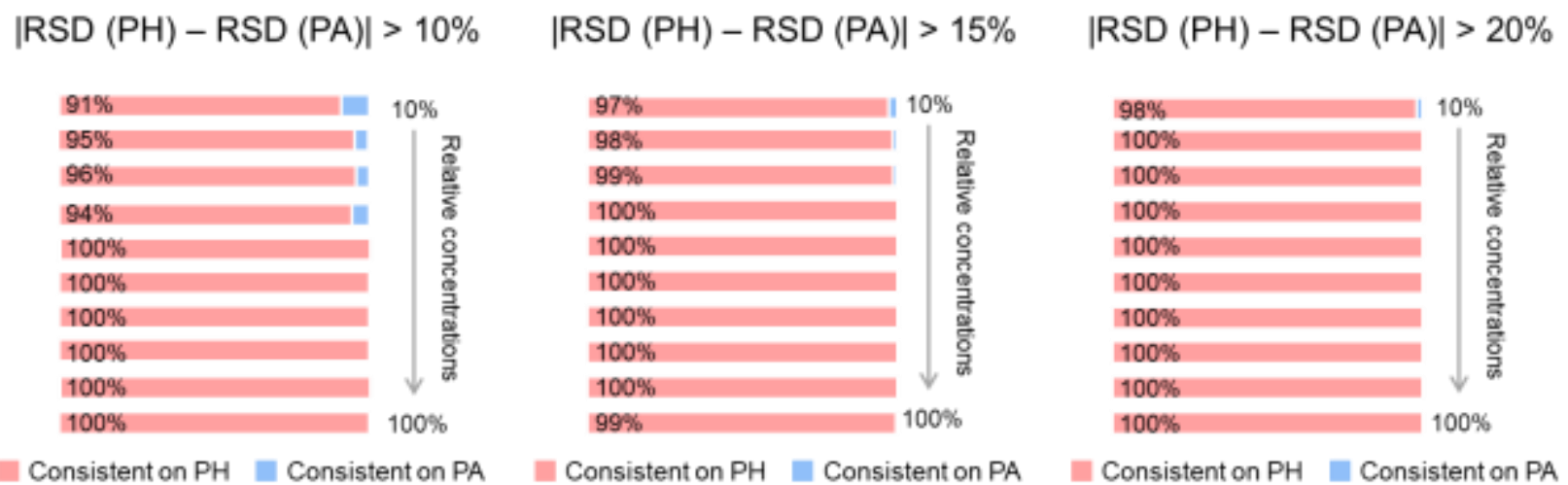

Figure S-10. Preference on peak height or peak area for metabolic features with consistent preference $(t=10,15,20 \%$, left to right). 


\section{A EIC of alpha-Ketoisovaleric acid}

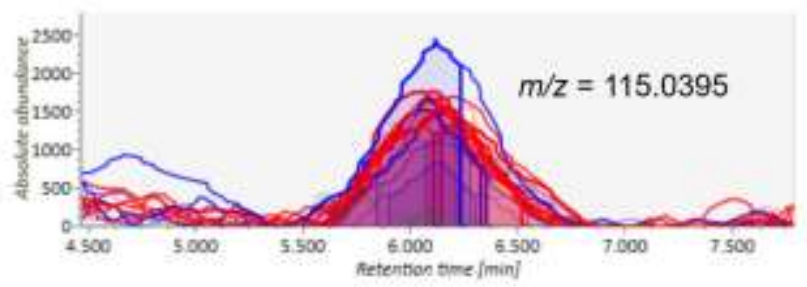

C

alpha-Ketoisovaleric acid
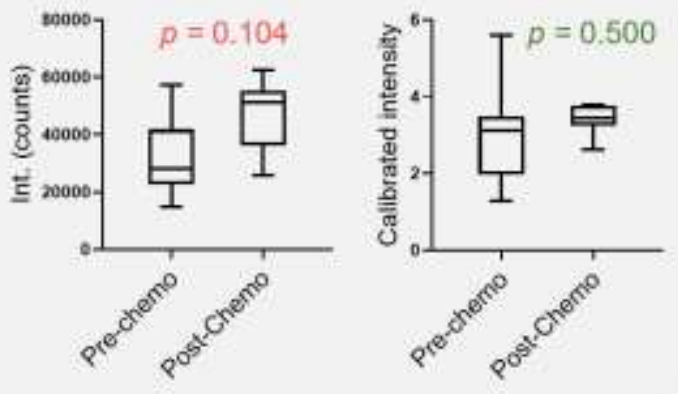

\begin{tabular}{|c|c|c|}
\hline B & $\begin{array}{c}\text { Before } \\
\text { Correction }\end{array}$ & $\begin{array}{c}\text { After } \\
\text { Correction }\end{array}$ \\
\hline Pre-chemo-1 & 28263 & 1.978 \\
\hline Pre-chemo-2 & 41909 & 3.117 \\
\hline Pre-chemo-3 & 22741 & 2.321 \\
\hline Pre-chemo-4 & 39645 & 3.136 \\
\hline Pre-chemo-5 & 14928 & 1.285 \\
\hline Pre-chemo-6 & 27663 & 3.485 \\
\hline Pre-chemo-7 & 57294 & 5.597 \\
\hline Post-chemo-1 & 38087 & 3.782 \\
\hline Post-chemo-2 & 25900 & 2.621 \\
\hline Post-chemo-3 & 36298 & 3.441 \\
\hline Post-chemo-4 & 55397 & 3.776 \\
\hline Post-chemo-5 & 51200 & 3.441 \\
\hline Post-chemo-6 & 52032 & 3.250 \\
\hline Post-chemo-7 & 62405 & 3.313 \\
\hline p-value & 0.104 & 0.500 \\
\hline
\end{tabular}

Figure S-11. A representative example of metabolite (alpha-Ketoisovaleric acid) with increased $p$-value after correction. (A) EIC of alpha-Ketoisovaleric acid $(\mathrm{m} / z=115.0395)$. The automatically integrated peak areas for the pre-chemo group was colored in blue and the post-chemo group in red. The meaning of bold and fine lines is same to the Figure S-9. (B) List of quantitative intensities for "before correction" and "after correction". (* wrong peak integration indicated; ** all 14 samples were selected to use the peak height-based quantitative information) (C) Box plot showed increased $p$-value after signal correction. 


\section{Significant Non-significant}
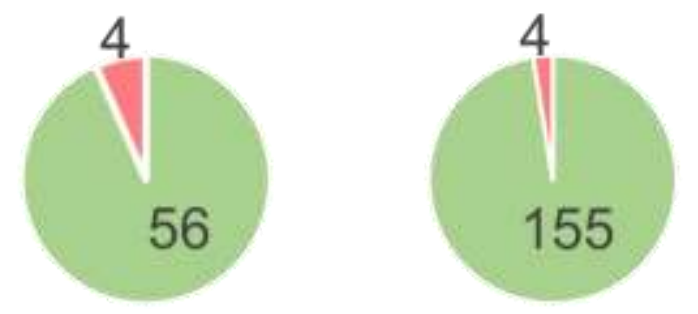

\section{Correct integration Poor integration}

Figure S-12. Manual inspection of the peak area-based quantitative results. Out of the 219 peak area-based quantitative results, 211 of them were corrected integrated, leading to a $96 \%$ correction rate. In addition, more metabolic features prefer peak area in "non-significant" group. This is because the correction result was compared to the purely peak area-based quantification method. Since "non-significant" means the $p$ value did not change significantly after correction, it make sense that more quantitative values prefer peak area. 
Table S-1. Concentration table of pooled 20 metabolite standard solution.

\begin{tabular}{|c|c|c|c|}
\hline Name & Retention time $(\mathrm{min})^{*}$ & Conc. $(\mu \mathrm{g} / \mathrm{mL})$ & Cat. No. $^{* *}$ \\
\hline DL-2-Aminoadipic acid & 11.27 & 50 & 52458 \\
\hline N-Acetyl-L-alanine & 8.11 & 81 & A4625 \\
\hline DL-Methionine sulfoxide & 10.40 & 46 & 64430 \\
\hline L-Carnosine & 11.38 & 60 & C9625 \\
\hline Adenosine 5'-diphosphoribose & 11.65 & 53 & A0752 \\
\hline 2'-Deoxycytidine & 8.16 & 55 & D3897 \\
\hline 4-Imidazoleacrylic acid & 9.40 & 15 & 859796 \\
\hline sn-Glycerol 3-phosphate & 11.67 & 68 & G7886 \\
\hline Creatinine & 7.26 & 60 & C4255 \\
\hline L-Alanine & 8.11 & 22 & AAS18 \\
\hline L-Arginine & 17.25 & 44 & AAS18 \\
\hline L-Aspartic acid & 11.40 & 33 & AAS18 \\
\hline Glycine & 10.74 & 19 & AAS18 \\
\hline Histidine & 10.84 & 39 & AAS18 \\
\hline L-Methionine & 9.22 & 37 & AAS18 \\
\hline L-Phenylalanine & 8.52 & 41 & AAS18 \\
\hline L-Proline & 9.52 & 29 & AAS18 \\
\hline L-Serine & 11.45 & 26 & AAS18 \\
\hline L-Tyrosine & 10.03 & 45 & AAS18 \\
\hline L-Valine & 9.55 & 29 & AAS18 \\
\hline & & & \\
\hline & & 29 & \\
\hline
\end{tabular}

* The retention times were obtained in HILIC negative ion mode.

** All metabolite standards were purchased from Sigma-Aldrich (St. Louis, MO). 
Table S-2. RSD comparison of using manual integration approach and automated computational approach in targeted LC-MS analysis.

\begin{tabular}{|c|c|c|c|c|}
\hline & $\begin{array}{c}\text { Peak height, } \\
\text { Manual } \\
\text { (RSD\%)* }\end{array}$ & $\begin{array}{c}\text { Peak height, } \\
\text { MS-DIAL } \\
\text { (RSD\%) }\end{array}$ & $\begin{array}{c}\text { Peak area, } \\
\text { Manual } \\
\text { (RSD\%) }\end{array}$ & $\begin{array}{c}\text { Peak area, } \\
\text { MS-DIAL } \\
\text { (RSD\% })\end{array}$ \\
\hline N-Acetyl-L-alanine & 0.62 & 1.23 & 1.18 & 1.34 \\
\hline L-Phenylalanine & 0.85 & 2.59 & 1.12 & 1.54 \\
\hline L-Arginine & 0.9 & 1.63 & 1.99 & 1.43 \\
\hline L-Alanine & 1.24 & 0.98 & 1.15 & 1.39 \\
\hline $\begin{array}{c}\text { Adenosine 5'- } \\
\text { diphosphoribose }\end{array}$ & 1.32 & 2.84 & 1.46 & 1.88 \\
\hline DL-2-Aminoadipic acid & 1.5 & 2.65 & 1.33 & 2.54 \\
\hline L-Valine & 2.24 & 2.14 & 2.29 & 3.14 \\
\hline 4-Imidazoleacrylic acid & 2.47 & 1.92 & 2.27 & 2.3 \\
\hline Creatinine & 2.53 & 2.71 & 2.06 & 1.94 \\
\hline L-Carnosine & 2.63 & 4.23 & 1.9 & 2.51 \\
\hline L-Serine & 2.75 & 2.94 & 0.51 & 1.07 \\
\hline 2'-Deoxycytidine & 2.79 & 3.27 & 2.44 & 2.56 \\
\hline sn-Glycerol 3-phosphate & 2.92 & 3.63 & 1.88 & 1.11 \\
\hline L-Aspartic acid & 3.16 & 5.67 & 2.41 & 4.16 \\
\hline L-Proline & 3.57 & 9.51 & 2.74 & 7.31 \\
\hline DL-Methionine sulfoxide & 4.24 & 2.54 & 1.82 & 1.9 \\
\hline Histidine & 4.31 & 7.31 & 1.37 & 1.77 \\
\hline L-Methionine & 4.47 & 2.52 & 1.38 & 1.35 \\
\hline Glycine & 6.41 & 7.2 & 2.48 & 3.33 \\
\hline L-Tyrosine & 7.39 & 8.57 & 3.7 & 4.51 \\
\hline Average: & 2.92 & 3.8 & 1.87 & 2.46 \\
\hline $\boldsymbol{p}$-value & & 0.03 & & 0.03 \\
\hline
\end{tabular}

* The table is sorted from low to high RSD using column "Peak height, Manual (RSD\%)". 
Table S-3. Number of extracted metabolic features* when $t=5 \%$.

\begin{tabular}{|c|c|c|c|}
\hline $\begin{array}{c}\text { Relative } \\
\text { concentration, \% }\end{array}$ & $\begin{array}{c}\text { \# of detected } \\
\text { features }\end{array}$ & $\begin{array}{c}\text { \# of extracted } \\
\text { features }\end{array}$ & $\begin{array}{c}\text { \# of agreed } \\
\text { features }\end{array}$ \\
\hline 10 & 780 & 213 & 136 \\
\hline 20 & 1053 & 252 & 163 \\
\hline 30 & 1232 & 230 & 148 \\
\hline 40 & 1433 & 231 & 140 \\
\hline 50 & 2126 & 385 & 251 \\
\hline 60 & 2321 & 389 & 248 \\
\hline 70 & 2293 & 396 & 265 \\
\hline 80 & 2399 & 396 & 265 \\
\hline 90 & 2536 & 443 & 287 \\
\hline 100 & 2814 & 539 & 343 \\
\hline
\end{tabular}

*: Certain threshold ( $\mathrm{t}$ value) of absolute RSD difference was used to extract metabolic features that have clear RSD differences in PA and PH-based quantifications. The meaning of extracted features is same in Tables S3-S6 
Table S-4. Number of extracted metabolic features when $t=10 \%$.

\begin{tabular}{|c|c|c|c|}
\hline $\begin{array}{c}\text { Relative } \\
\text { concentration, \% }\end{array}$ & $\begin{array}{c}\text { \# of detected } \\
\text { features }\end{array}$ & $\begin{array}{c}\text { \# of extracted } \\
\text { features }\end{array}$ & $\begin{array}{c}\text { \# of agreed } \\
\text { features }\end{array}$ \\
\hline 10 & 780 & 114 & 87 \\
\hline 20 & 1053 & 116 & 88 \\
\hline 30 & 1232 & 125 & 92 \\
\hline 40 & 1433 & 127 & 88 \\
\hline 50 & 2126 & 226 & 179 \\
\hline 60 & 2321 & 235 & 185 \\
\hline 70 & 2293 & 254 & 203 \\
\hline 80 & 2399 & 253 & 201 \\
\hline 90 & 2536 & 297 & 222 \\
\hline 100 & 2814 & 347 & 262 \\
\hline
\end{tabular}


Table S-5. Number of extracted metabolic features when $t=15 \%$.

\begin{tabular}{|c|c|c|c|}
\hline $\begin{array}{c}\text { Relative } \\
\text { concentration, \% }\end{array}$ & $\begin{array}{c}\text { \# of detected } \\
\text { features }\end{array}$ & $\begin{array}{c}\text { \# of extracted } \\
\text { features }\end{array}$ & $\begin{array}{c}\text { \# of agreed } \\
\text { features }\end{array}$ \\
\hline 10 & 780 & 83 & 68 \\
\hline 20 & 1053 & 72 & 61 \\
\hline 30 & 1232 & 81 & 68 \\
\hline 40 & 1433 & 77 & 59 \\
\hline 50 & 2126 & 151 & 126 \\
\hline 60 & 2321 & 152 & 125 \\
\hline 70 & 2293 & 162 & 141 \\
\hline 80 & 2399 & 168 & 145 \\
\hline 90 & 2536 & 190 & 161 \\
\hline 100 & 2814 & 226 & 190 \\
\hline
\end{tabular}


Table S-6. Number of extracted metabolic features when $t=20 \%$.

\begin{tabular}{|c|c|c|c|}
\hline $\begin{array}{c}\text { Relative } \\
\text { concentration, \% }\end{array}$ & $\begin{array}{c}\text { \# of detected } \\
\text { features }\end{array}$ & $\begin{array}{c}\text { \# of extracted } \\
\text { features }\end{array}$ & $\begin{array}{c}\text { \# of agreed } \\
\text { features }\end{array}$ \\
\hline 10 & 780 & 59 & 49 \\
\hline 20 & 1053 & 47 & 42 \\
\hline 30 & 1232 & 52 & 45 \\
\hline 40 & 1433 & 43 & 36 \\
\hline 50 & 2126 & 83 & 74 \\
\hline 60 & 2321 & 88 & 77 \\
\hline 70 & 2293 & 94 & 85 \\
\hline 80 & 2399 & 94 & 86 \\
\hline 90 & 2536 & 123 & 111 \\
\hline 100 & 2814 & 134 & 117 \\
\hline
\end{tabular}


Text S-1. Metabolite extraction of biological samples.

1) Polar metabolite extraction of human serum, urine, and BMIF samples

A $50 \mu \mathrm{L}$ serum sample was mixed with $10 \mu \mathrm{L}$ internal standard mixture (D-Glucose- ${ }^{13} \mathrm{C}_{6}(11.8$

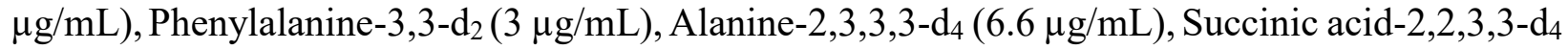
$(6.2 \mu \mathrm{g} / \mathrm{mL})$, and Lysine-4,4,5,5- $\left.\mathrm{d}_{4}(6.4 \mu \mathrm{g} / \mathrm{mL})\right)$ and $1 \mathrm{~mL}$ ice-cold methanol in a $1.5 \mathrm{~mL}$ Eppendorf vial. After 2 min vortex, the solution was incubated at $-20{ }^{\circ} \mathrm{C}$ for $2 \mathrm{~h}$ to precipitate proteins, followed by centrifugation at $14,000 \mathrm{rpm}$ at $4{ }^{\circ} \mathrm{C}$ for $15 \mathrm{~min}$. The clear supernatant was dried in a SpeedVac at $20{ }^{\circ} \mathrm{C}$ and then reconstituted in $150 \mu \mathrm{L}$ ACN and water $(1: 1, \mathrm{v}: \mathrm{v})$ mixed solvent for LC-MS analysis (high concentration solution). The human serum sample with low concentration was a 100-fold dilution of the high concentration solution. In addition, to test the contribution of reconstitution solution composition on computational variation, another dried metabolome extract of the same serum was reconstituted in $150 \mu \mathrm{L}$ ACN and water (9:1, v:v) mixed solvent for LC-MS analysis. The method blank was also prepared following the same protocol but without serum.

Similar to serum sample preparation, $100 \mu \mathrm{L}$ of urine sample was used and reconstituted to 100 $\mu \mathrm{L}$. All other procedures of urine metabolome extraction are the same as the human serum sample demonstrated above.

In addition, $10 \mathrm{mg}$ of mouse fecal sample and $10 \mathrm{mg}$ of mouse gut content sample was used for metabolome extraction. $1 \mathrm{~mL}$ of ice-cold $\mathrm{MeOH} /$ water $(9: 1, \mathrm{v} / \mathrm{v})$ was added to each sample followed by sonication in ice-bath for $30 \mathrm{~min}$. After $2 \mathrm{~min}$ vortex, the solution was incubated at $-20{ }^{\circ} \mathrm{C}$ for $4 \mathrm{~h}$ to precipitate proteins, followed by centrifugation at $14,000 \mathrm{rpm}$ at $4{ }^{\circ} \mathrm{C}$ for $15 \mathrm{~min}$. 
The clear supernatant was processed using the same procedure for human serum sample. Method blank sample was also prepared following the same protocol but without fecal or gut content sample.

The procedure of BMIF metabolome extraction is identical to the human serum sample demonstrated above.

2) Nonpolar lipid extraction of human serum sample

A $50 \mu \mathrm{L}$ serum sample was mixed with $270 \mu \mathrm{L}$ ice-cold methanol in a $1.5 \mathrm{~mL}$ Eppendorf vial. After $2 \mathrm{~min}$ vortex, the solution was incubated at $-20^{\circ} \mathrm{C}$ for $2 \mathrm{~h}$ to precipitate proteins, followed by adding $900 \mu \mathrm{L}$ methyl tert-butyl ether (MTBE) for lipid extraction. The one-phase solvent was shaken for $2 \mathrm{~min}$ and $265 \mu \mathrm{L}$ of water was added to induce the phase separation. After $15 \mathrm{~s}$ vortex, the solution rested at room temperature for $10 \mathrm{~min}$. Next, the solution was centrifuged at 14,000 rpm for 15 min at $4{ }^{\circ} \mathrm{C}$. The clear upper layer was separated into a new vial and dried in a SpeedVac at $20{ }^{\circ} \mathrm{C}$. Then, the dried extract was reconstituted in $150 \mu \mathrm{L}$ ACN and isopropanol (1:1, v:v) mixed solvent for LC-MS analysis (high concentration solution). The low concentration solution was a 100-fold dilution of the high concentration solution. The method blank was also prepared following the same protocol but without adding serum. 
Text S-2. LC gradient and other instrumental settings.

1) LC separation on HILIC column of polar metabolites

Mobile phase A was 5\% ACN in water with $10 \mathrm{mM}$ ammonium acetate (pH 9.8), and mobile phase B was $95 \% \mathrm{ACN}$ in water with no buffer. The flow rate was $0.15 \mathrm{~mL} / \mathrm{min}$. The LC gradient was as follows: $0 \mathrm{~min}, 95 \% \mathrm{~B} ; 20 \mathrm{~min}, 5 \% \mathrm{~B} ; 25 \mathrm{~min}, 5 \% \mathrm{~B} ; 26 \mathrm{~min}, 95 \% \mathrm{~B} ; 40 \mathrm{~min}, 95 \% \mathrm{~B}$. The column temperature was set at $25^{\circ} \mathrm{C}$ and the injection volumes of human serum and urine samples were $5 \mu \mathrm{L}$. The injection volume of BMIF samples was optimized to $5 \mu \mathrm{L}$ using QC sample.

2) LC separation on reverse phase C18 column of polar metabolites

Mobile phase A was water with $0.1 \%$ formic acid, and mobile phase B was ACN with $0.1 \%$ formic acid. The flow rate was $0.15 \mathrm{~mL} / \mathrm{min}$. The LC gradient was as follows: $0 \mathrm{~min}, 5 \% \mathrm{~B} ; 8 \mathrm{~min}, 25 \%$ B; $14 \min , 70 \% \mathrm{~B} ; 20 \mathrm{~min}, 95 \% \mathrm{~B} ; 23 \mathrm{~min}, 95 \% \mathrm{~B} ; 23.01 \mathrm{~min}, 5 \% \mathrm{~B} ; 30 \mathrm{~min}, 5 \% \mathrm{~B}$. The column temperature was set at $25^{\circ} \mathrm{C}$ and the injection volumes of human serum and urine samples were 5 $\mu \mathrm{L}$.

3) LC separation on reverse phase C18 column of nonpolar lipids

Mobile phase A was ACN and $\mathrm{H}_{2} \mathrm{O}(6: 4, \mathrm{v}: \mathrm{v})$, and mobile phase B was IPA and ACN (9:1, v:v). Both mobile phases contain $0.1 \%$ formic acid (FA) and $5 \mathrm{mM}$ ammonium formate $\left(\mathrm{NH}_{4} \mathrm{FA}\right)(\mathrm{pH}$ = 4.8). The flow rate was $0.10 \mathrm{~mL} / \mathrm{min}$. The LC gradient was set as follows: $0 \mathrm{~min}, 5 \% \mathrm{~B} ; 8 \mathrm{~min}$, $40 \% \mathrm{~B} ; 14 \mathrm{~min}, 70 \% \mathrm{~B} ; 20 \mathrm{~min}, 95 \% \mathrm{~B} ; 23 \mathrm{~min}, 95 \% \mathrm{~B} ; 24 \mathrm{~min}, 5 \% \mathrm{~B} ; 33 \mathrm{~min}, 5 \% \mathrm{~B}$. The column temperature was set at $25^{\circ} \mathrm{C}$ and the injection volume of human serum lipidome extract was $3 \mu \mathrm{L}$. 
Text S-3. MS parameter settings.

1) ESI negative ion mode in data dependent acquisition mode for polar metabolites

Capillary voltage, $3.0 \mathrm{kV}$; nebulizer gas, $1.6 \mathrm{bar}$; dry gas, $7 \mathrm{~L} / \mathrm{min}$; dry gas temperature, $220{ }^{\circ} \mathrm{C}$; mass scan range, 65-1500 ( $\mathrm{m} / \mathrm{z})$; spectra rate, $8.00 \mathrm{~Hz}$; and cycle time, 3.0 s. For centroid spectra calculation, the peak summation width was 3 pts. The mass spectrometer was calibrated using sodium formate (NaFA, $250 \mathrm{mM}$ ) before analysis.

2) ESI positive ion mode in data dependent acquisition mode for polar metabolites Capillary voltage, $4.5 \mathrm{kV}$; nebulizer gas, 1.6 bar; dry gas, $7 \mathrm{~L} / \mathrm{min}$; dry gas temperature, $220{ }^{\circ} \mathrm{C}$; mass scan range, 70-1020 (m/z); spectra rate, $8.00 \mathrm{~Hz}$; and cycle time, 3.0 s. For centroid spectra calculation, the peak summation width was 3 pts. The mass spectrometer was calibrated using NaFA (250 mM) before analysis.

3) ESI positive ion mode in data dependent acquisition mode for nonpolar lipids Capillary voltage, $4.5 \mathrm{kV}$; nebulizer gas, 1.0 bar; dry gas, $6 \mathrm{~L} / \mathrm{min}$; dry gas temperature, $220{ }^{\circ} \mathrm{C}$; mass scan range, 50-2500 ( $\mathrm{m} / \mathrm{z})$; spectra rate, $8.00 \mathrm{~Hz}$; and cycle time, $3.0 \mathrm{~s}$. For centroid spectra calculation, the peak summation width was 3 pts. The mass spectrometer was calibrated using NaFA (250 mM) before analysis. 
Text S-4. MS-DIAL parameter settings for data processing.

Mass accuracy

$\mathrm{MS}^{1}$ tolerance: $0.01, \mathrm{MS}^{2}$ tolerance: 0.05

Peak detection

Minimum peak height: 1000 amplitude

Mass slice width: $0.05 \mathrm{Da}$

\section{Identification}

MSMS library: http://prime.psc.riken.jp/compms/msdial/main.html

Accurate mass tolerance $\left(\mathrm{MS}^{1}\right): 0.01$

Accurate mass tolerance $\left(\mathrm{MS}^{2}\right): 0.05$

The retention time is not used for scoring.

\section{Adduct}

Positive ion mode: $[\mathrm{M}+\mathrm{H}]^{+},\left[\mathrm{M}-\mathrm{H}_{2} \mathrm{O}+\mathrm{H}\right]^{+},\left[\mathrm{M}+\mathrm{NH}_{4}\right]^{+}$

Negative ion mode: $[\mathrm{M}-\mathrm{H}]^{-},\left[\mathrm{M}-\mathrm{H}_{2} \mathrm{O}-\mathrm{H}\right]^{-}$

\section{Alignment}

Retention time tolerance: $0.3 \mathrm{~min}$

$\mathrm{MS}^{1}$ tolerance: $0.01 \mathrm{Da}$ 
Text S-5. XCMS parameter settings for data processing (optimized).

The XCMS centWave algorithm was utilized for peak detection.

$\mathrm{ppm}=10$,

peakwidth $=(10,60)$

$\mathrm{m} / \mathrm{z} \operatorname{diff}=0.01$,

snthresh $=6$,

integrate $=1$,

prefilter $=(3,100)$,

noise $=100$

$\mathrm{bw}=5$

mzwid $=0.015$

minsamp $=1, \max =50$ 
Text S-6. XCMS parameter settings for data processing (not optimized).

The XCMS centWave algorithm was utilized for peak detection.

ppm $=10$,

peakwidth $=(5,100)$

$\mathrm{m} / \mathrm{z} \operatorname{diff}=0.01$,

snthresh $=6$,

integrate $=1$,

prefilter $=(3,100)$,

noise $=100$

$\mathrm{bw}=5$

mzwid $=0.015$

minsamp $=1, \max =100$ 
Text S-7. Manual inspection in MS-DIAL.

In brief, we determined "correct integration by checking the MS-DIAL results. After extracting the metabolic feature table (see the upper picture), we double clicked the chromatogram column to view a zoom-in page of peak area integration as shown in the bottom picture. The area under the EIC curve was manually investigated by a well-trained MS expert. In particular, correct integration means the integration by software is same or very similar to what we expected to be correct integration.

\begin{tabular}{|c|c|c|c|c|c|c|c|c|c|c|c|}
\hline \multicolumn{11}{|c|}{ 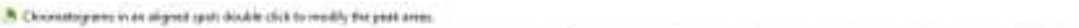 } & \multirow{2}{*}{$\frac{-\square}{\text { Crimungum }}$} \\
\hline$\neq 0$ & Fithene & then in & $a=$ & twaukn & Tousene & m & a & sh: & Panim & Baselner & \\
\hline 8 & fastagengly & $w$ & setrr & & 100 & inar & in & mis & nim & Tise & \\
\hline T & itecanen & 酷 & $=$ & & (ino & mat & isi & wes & tim & Dant & \\
\hline$x^{\prime}$ & imataven & $\equiv$ & setser & & 1010 & sean & $\mathrm{G}_{4}$ & काA & Tin & Ev04t & \\
\hline T & wondery & tifi & $\operatorname{sinm}$ & & $-7 \infty$ & $m \infty$ & in & win & $m$ & awn & \\
\hline ? & movedifos & 4 & sinter & & 10 & sका & 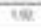 & 716.3 & किसा & 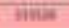 & \\
\hline 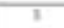 & 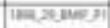 & 101 & Athe & & -150 & Inise & $\tan$ & 31 & $\operatorname{In} 4$ & पа木ы & \\
\hline 7 & waven & \$) & Shan & & -100 & Indi & एवi & का & पive & Jaing & \\
\hline$T$ & inasuent & a & Whe & & 400 & Inen & II & $m$ & ज़ा & bente & \\
\hline 7 & 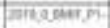 & $=$ & Fists & & 100 & ming & (a) & क्य & प्ता & क्षका & \\
\hline 7 & meaver & 148 & Nat & & 100 & $\min$ & $\omega$ & $=m$ & $\tan$ & ज्ञात & \\
\hline 7 & incopoim & 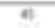 & stor & & 100 & 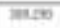 & ex. & if & nte & beent: & \\
\hline$\pi$ & An: & 194 & ant & & 10 & $\operatorname{sen} 2$ & राi & का & Sile & Weiz & \\
\hline ut & 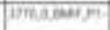 & का & $\sin$ & & $10 x$ & $\operatorname{sen}$ & init & का & sivin & thets & $A$ \\
\hline 11 & 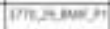 & $=$ & Antr & & $1 \mathrm{~m}$ & mam & in & in: & कासा & नात & \\
\hline
\end{tabular}

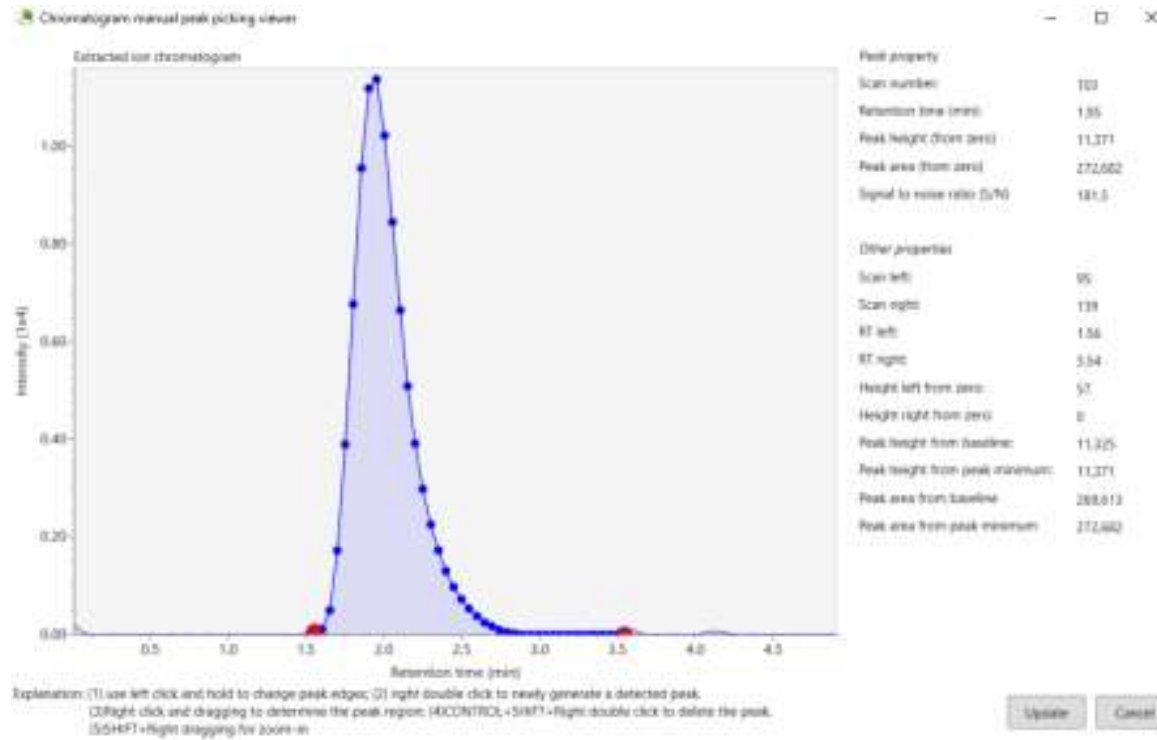

